\title{
Mild Zellweger syndrome due to functionally confirmed novel PEX1 variants
}

\author{
Patryk Lipiński ${ }^{1}$ - Piotr Stawiński ${ }^{2}$ Małgorzata Rydzanicz ${ }^{2} \cdot$ Maria Wypchło $^{2,3} \cdot$ Rafał Płoski $^{2}$. \\ Teresa Joanna Stradomska ${ }^{4}$ Elżbieta Jurkiewicz ${ }^{5} \cdot$ Sacha Ferdinandusse $^{6} \cdot$ Ronald J. A. Wanders $^{6} \cdot$ Frederic M. Vaz $^{6}$. \\ Anna Tylki-Szymańska ${ }^{1}$
}

Received: 26 April 2019 /Revised: 31 August 2019 / Accepted: 11 September 2019 / Published online: 18 October 2019

(C) The Author(s) 2019

\begin{abstract}
Zellweger spectrum disorders (ZSD) constitute a group of rare autosomal recessive disorders characterized by a defect in peroxisome biogenesis due to mutations in one of 13 PEX genes. The broad clinical heterogeneity especially in late-onset presenting patients and a mild phenotype complicates and delays the diagnostic process. Here, we report a case of mild ZSD, due to novel $P E X 1$ variants. The patient presented with an early hearing loss, bilateral cataracts, and leukodystrophy on magnetic resonance (MR) images. Normal results of serum very-long-chain fatty acids (VLCFA) and phytanic acid were found. Molecular diagnostics were performed to uncover the etiology of the clinical phenotype. Using whole exome sequencing, there have been found two variants in the PEX1 gene-c.3450T>A (p.Cys1150*) and c.1769T>C (p.Leu590Pro). VLCFA measurement in skin fibroblasts and C26:0-lysoPC in dried blood spot therefore was performed. Both results were in line with the diagnosis of ZSD. To conclude, normal results of routine serum VLCFA and branched-chain fatty acid measurement do not exclude mild forms of ZSD. The investigation of $\mathrm{C} 26: 0-1 y s o P C$ should be included in the diagnostic work-up in patients with cataract, hearing loss, and leukodystrophy on MR images suspected to suffer from ZSD.
\end{abstract}

Keywords Zellweger spectrum disorders $\cdot$ PEX1 gene $\cdot$ C26:0-lysoPC

\begin{tabular}{|c|c|c|}
\hline Abbreviations & & DBS \\
\hline C26:0-lysoPC & C26:0-Lysophosphatidylcholine & MR \\
\hline $\mathrm{CT}$ & Computed tomography & NGS \\
\hline Communicated by & Michal Witt & \\
\hline
\end{tabular}

Anna Tylki-Szymańska

a.tylki@ipczd.pl

1 Department of Pediatrics, Nutrition and Metabolic Disease, The Children's Memorial Health Institute, Al. Dzieci Polskich 20, 04-730 Warsaw, Poland

2 Department of Medical Genetics, Medical University of Warsaw, Warsaw, Poland

3 Postgraduate School of Molecular Medicine, Medical University of Warsaw, Warsaw, Poland

4 Department of Biochemistry, Radioimmunology and Experimental Medicine, The Children's Memorial Health Institute, Warsaw, Poland

5 Department of Diagnostic Imaging, The Children's Memorial Health Institute, Warsaw, Poland

6 Laboratory Genetic Metabolic Diseases, Academic Medical Center, University of Amsterdam, Amsterdam, The Netherlands

\author{
Dried blood spot \\ Magnetic resonance \\ Next-generation sequencing \\ Very-long-chain fatty acids \\ Whole exome sequencing \\ Zellweger spectrum disorders
}

\section{Introduction}

Zellweger spectrum disorders constitute a group of rare autosomal recessive disorders characterized by a defect in peroxisome biogenesis due to mutations in one of 13 PEX genes (Klouwer et al. 2015). The broad clinical heterogeneity especially in late-onset presenting patients and a mild phenotype complicates and delays the diagnostic process (Klouwer et al. 2016; Berendse et al. 2016).

Here, we report a patient diagnosed with ZSD at the age of 21 presenting with early hearing loss and then bilateral cataracts and leukodystrophy with normal results of standard biochemical markers (including serum very-long-chain fatty 
acids (VLCFA)), in whom the molecular analysis (whole exome sequencing (WES)) prompted to investigation of $\mathrm{C} 26: 0$ lysoPC in DBS and VLCFA in skin fibroblasts to confirm the diagnosis.

\section{Case report}

The patient was the third child of nonconsanguineous Polish parents, born at term from an uncomplicated pregnancy with a body mass of $3100 \mathrm{~g}$. During the first year of life, he had undergone two episodes of pneumonia; during the first episode, a vitamin $\mathrm{K}$-dependent coagulopathy was diagnosed. The laboratory analyses revealed anemia and slightly elevated liver transaminases (Table 1). At the age of 1.5 years, nystagmus was noted. Liver transaminases had normalized but the prothrombin time was still prolonged.

The patient's psychomotor development was delayed; he was able to walk alone at the age of 3.5 years. From the age of 5 years, he received a hearing aid because of hypoacusis. At the age of 12 years, a progressive spastic paresis was diagnosed. A mild intellectual disability and slowing head circumference growth were also observed. At the age of 18 , bilateral cataracts were noted. CT brain imaging done at that age revealed mild generalized cortical and subcortical atrophy.

The family history revealed that patient's older sister had presented with similar signs including more pronounced intellectual disability, bilateral hearing loss, and cataracts but no diagnosis was established.

The patient became completely wheelchair dependence when at 21 years old. Due to progressive mental retardation, magnetic resonance (MR) imaging was done and the brain T2weighted images (see Fig. 1) showed cerebral white matter hyperintensity in the both centrum semiovale posteriorly with sparing of subcortical fibers, bilateral areas of signal increase surrounding posterior parts of lateral ventricles, hyperintensive posterior limbs of the internal capsules, and the small hyperintense signal in the genu and splenium of the corpus callosum. Infratentorially, the signal increased in the central cerebellar white matter area and in the hilus of the dentate nucleus, bilaterally. Dentate nucleus between hilus and central cerebellar white matter were spared.

Table 1 The patient's signs and symptoms and biochemical and molecular outcome

\begin{tabular}{|c|c|c|}
\hline Age & Symptoms and signs & Results of laboratory tests/imaging procedures \\
\hline First year of life & $\begin{array}{l}2 \text { episodes of pneumonia; vitamin } \mathrm{K} \text {-dependent } \\
\text { coagulopathy }\end{array}$ & $\begin{array}{l}\text { Elevated liver transaminases (AST 109,74; ALT 65,75), prolonged } \\
\text { PT 17.2/15.4, normal results of metabolic screening tests }{ }^{\#}\end{array}$ \\
\hline $1.5 \mathrm{y}$ & Third episode of pneumonia; nystagmus & $\begin{array}{l}\text { Normal liver transaminases, prolonged PT } 18.5 \text {, normal results } \\
\text { of metabolic screening tests }\end{array}$ \\
\hline $4 \mathrm{y}$ & Delayed psychomotor development; hearing loss & n.a. \\
\hline $12 \mathrm{y}$ & $\begin{array}{l}\text { Partial wheelchair dependency due to spastic } \\
\text { paresis; mild intellectual disability; slowing } \\
\text { head circumference growth }\end{array}$ & n.a. \\
\hline $18 \mathrm{y}$ & Bilateral cataracts & $\begin{array}{l}\text { Normal liver transaminases, normal PT, normal results of metabolic } \\
\text { screening tests, CT imaging-mild generalized cortical and } \\
\text { subcortical atrophy }\end{array}$ \\
\hline $21 \mathrm{y}$ & $\begin{array}{l}\text { Total wheelchair dependency; anger attacks } \\
\text { with progressive mental retardation; } \\
\text { microcephaly }\end{array}$ & $\begin{array}{l}\text { Normal results of metabolic screening tests, } \\
{ }^{*} \mathrm{C} 24: 0 / \mathrm{C} 220-0.910 ; N<0.96 \\
\mathrm{C} 26: 0 / \mathrm{C} 220-0.015 ; N<0.02 \\
\text { Phytanic acid }(\mu \mathrm{g} / \mathrm{mL})-0.37 ; N<3.3 \\
* * \mathrm{C} 24: 0 / \mathrm{C} 220-0.940 ; N<0.96 \\
\mathrm{C} 26: 0 / \mathrm{C} 220-0.020 ; N<0.02 \\
\text { Phytanic acid }(\mu \mathrm{g} / \mathrm{mL})-0.47 ; N<3.3 \\
\text { VLCFA in skin fibroblasts } \\
\text { C24:0/C22 } 0-4.09 ; N=1.68-2.92 \\
\text { C26:0/C22 } 0-0.92 ; N=0.03-0.10 \\
\text { C26:0-lysoPC (pmol/mg protein)-131; } N=2-14 \\
\text { Leukodystrophy on MR imaging (see Fig. } 1 \text { ) }\end{array}$ \\
\hline
\end{tabular}

mo months, $y$ years, n.a. not analyzed, CT computed tomography, MR magnetic resonance, ALT alanine aminotransferase (U/1), AST aspartate aminotransferase (U/l), $P T$ prothrombin time (s)

Reference values: ALT, $<18$ months, $<55 / 60 \mathrm{U} / \mathrm{L} ; 18$ months-12 years, boys, $<40 \mathrm{U} / \mathrm{L}$, girls, $<35 \mathrm{U} / \mathrm{L} ;>12$ years, boys, <26 U/L, girls, <22 U/L; AST, $<52 \mathrm{U} / \mathrm{L}$

*1st measurement

$* * 2$ nd measurement

${ }^{\#} \mathrm{GC} / \mathrm{MS}$ analysis of organic acids in urine, tandem mass spectrometry analysis (MS/MS), transferrin isoforms analysis, serum ammonia and lactate, serum VLCFA, urine and serum arabitol, urinary glycosaminoglycans analysis 


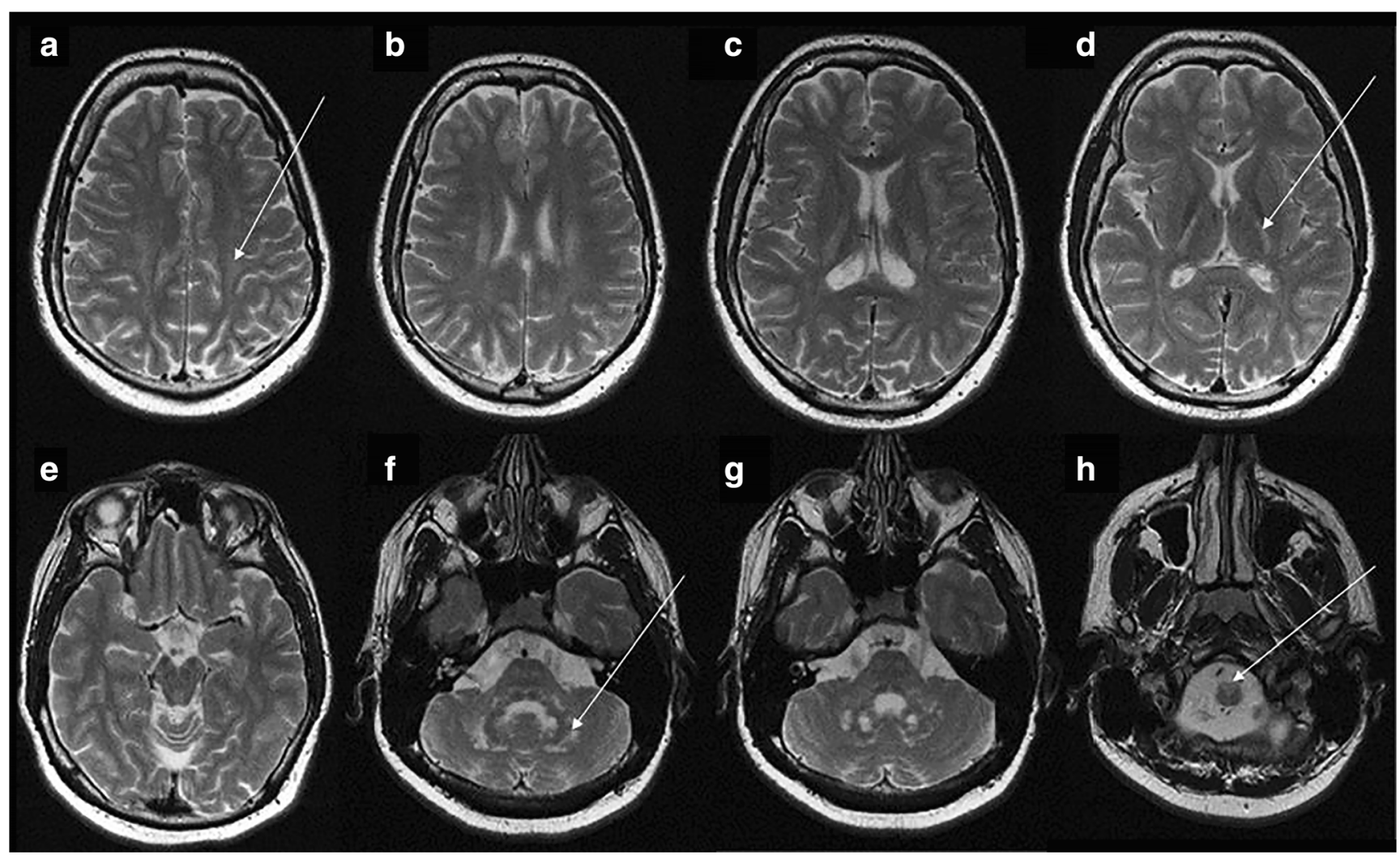

Fig. 1 MRI examination at the age of 21 years. Axial T2-weighted images. See the bilateral hyperintense signal of the posterior part of the centrum semiovale (arrow on a), posterior limb of internal capsule (arrow on d), cerebellar white matter (arrow on $\mathbf{f}$ ). The antero-medial part of medulla oblongata is also hyperintesive (arrow on $\mathbf{h}$ )

prediction scores from SIFT, PolyPhen-2, GERP++, MutationTaster, Mutation Assessor, FATHMM, LRT, SiPhy, and PhyloP) (https://varsome.com). Both variants have not been reported in GnomAD (http://gnomad.broadinstitute. org) or in our own database including over 1000 WES samples from a Polish population.

Thus, based on WES analysis, we found two novel potentially damaging $P E X 1$ variants which occurred in trans in both affected sibs consistent with autosomal recessive inheritance.

In order to determine whether these variants had affected enzymatic function, biochemical examinations were performed as described elsewhere (Stradomska and TylkiSzymańska 1996; Takemoto et al. 2003). Surprisingly, we found normal results of serum VLCFA and phytanic acid (Table 1). We therefore continued and performed VLCFA measurement in skin fibroblasts and $\mathrm{C} 26: 0-1 y s o P C$ in dried blood spot as described by Klouwer et al. (2017) in the Laboratory of Genetic Metabolic Diseases in Amsterdam. Both results (Table 1) were in line with the diagnosis of ZSD.

\section{Discussion}

The paper presents diagnostic difficulties in establishing a diagnosis of ZSD in a 21-year-old patient based on 
a

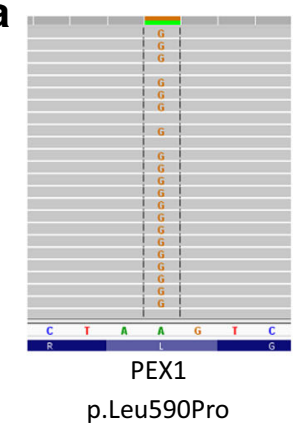

hg19:chr7:092136342-A>G

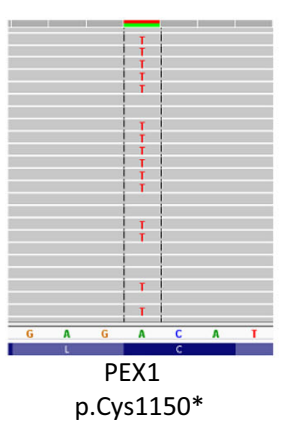

hg:19:chr7:092119214-A>T

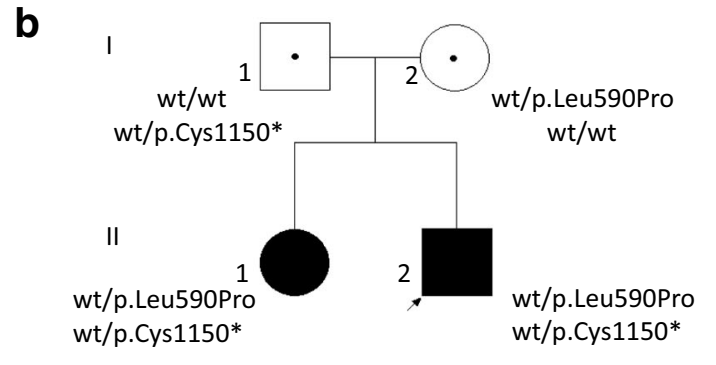

Fig. 2 a WES results in the proband-view from Integrative Genomics Viewer (IGV). b Pedigree of the family with phenotype/genotype information. The proband is indicated with black arrow (wt wild type).

standard biochemical markers, including serum VLCFA and phytanic acid.

Nowadays, next-generation sequencing techniques including gene panels or event whole exome sequencing are new possibilities to identify the molecular background of diseases with an unknown etiology. Because of this, there are an increasing number of patients with a mild phenotype of ZSD initially diagnosed by molecular analysis (Ebberink et al. 2010; Ebberink et al. 2012; Sevin et al. 2011; Ventura et al. 2016; Rydzanicz et al. 2017). The molecular results, however, should always be interpreted in combination with the clinical phenotype and appropriate laboratory analyses. The presented case and also findings from other reports supports the thesis that the level of serum markers of peroxisome dysfunction may not reflect the level of tissue accumulation (Berendse et al. 2016; Sevin et al. 2011). As in our previous report, VLCFA levels in patients presenting with a mild phenotype are generally below the values characteristic for patients with classical severe disease (Rydzanicz et al. 2017).

The standard biochemical serum markers of peroxisomes dysfunction include accumulation of VLCFA, phytanic and pristanic acid, and C27-bile acid intermediates and a deficiency of erythrocyte plasmalogens (Stradomska and TylkiSzymańska 2009; Wanders et al. 2017). A positive correlation of $\mathrm{C} 26: 0$ and $\mathrm{C} 26: 0 / \mathrm{C} 22: 0$ ratio with VLCFA accumulation has been shown (Berendse et al. 2016; Stradomska and TylkiSzymańska 2009; Raas-Rothschild et al. 2002; Tran et al. c Graphical presentation of Sanger sequencing verification of detected PEX1 variants in the proband and her family - view from Variant Reporter 1.1

2014); however, in some patients, especially those with a mild phenotype or affected with certain $P E X$ genes mutations, serum VLCFA can be completely normal (Berendse et al. 2016; Ebberink et al. 2010; Sevin et al. 2011). Normalization of serum VLCFA with age has been also reported (Berendse et al. 2016). Therefore, there is a need of better biochemical markers in diagnostic work-up in patients suspected to suffer from ZSD. Recently, Klouwer et al. commenced a novel serum biomarker, C26:0-lysoPC, and it has been reported as a sensitive marker in ZSD patients, more sensitive than other biochemical markers such as serum VLCFA and phytanic acid (Klouwer et al. 2017). Yet, even if all test results are normal in plasma and urine, but a high level of suspicion for a ZSD remains, additional testing in fibroblasts should be considered to definitely rule out a peroxisomal disorder (Klouwer et al. 2016).

Based on the occurrence of early hearing loss and then bilateral cataracts and leukodystrophy on MR images, we could retrospectively categorize patient to be suffered from mild ZSD based on the severity scoring scale proposed by Klouwer et al. (Klouwer et al. 2018). As in our previously published case and report of Weller et al. (Weller et al. 2008), the leukoencephalopathy and brain atrophy are the commonest changes found in mildly affected patients. This case supports also the hypothesis termed by Klouwer et al. about eye abnormalities (especially cataract) and hearing loss as the key symptoms in ZSD. 


\section{Conclusions}

Normal results of routine serum VLCFA and branched-chain fatty acid measurement do not exclude mild forms of ZSD.

The investigation of C26:0-lysoPC should be included in the diagnostic work-up in patients with hearing loss, cataract, progressive intellectual disability, spastic paraparesis, and leukodystrophy on MR images suspected to suffer from ZSD.

Acknowledgments The authors would like to thank the Polish Association of Patients with Mucopolysaccharidosis and Rare Diseases, for granting the funding on WES analysis.

Funding information WES analysis was funded by the Polish Association of Patients with Mucopolysaccharidosis and Rare Diseases.

\section{Compliance with ethical standards}

Ethical approval All procedures performed in studies involving human participants were in accordance with the ethical standards of the institutional and/or national research committee and with the 1964 Helsinki declaration and its later amendments or comparable ethical standards.

Conflict of interest The authors declare that they have no conflict of interest.

Open Access This article is distributed under the terms of the Creative Commons Attribution 4.0 International License (http:// creativecommons.org/licenses/by/4.0/), which permits unrestricted use, distribution, and reproduction in any medium, provided you give appropriate credit to the original author(s) and the source, provide a link to the Creative Commons license, and indicate if changes were made.

\section{References}

Berendse K, Engelen M, Ferdinandusse S et al (2016) Zellweger spectrum disorders: clinical manifestations in patients surviving into adulthood. J Inherit Metab Dis 39:93-106

Ebberink MS, Csanyi B, Chong WK et al (2010) Identification of an unusual variant peroxisome biogenesis disorder caused by mutations in the PEX16 gene. J Med Genet 47:608-615

Ebberink MS, Koster J, Visser G et al (2012) A novel defect of peroxisome division due to a homozygous non-sense mutation in the PEX11 $\beta$ gene. J Med Genet 49:307-313
Klouwer FCC, Berendse K, Ferdinandusse S et al (2015) Zellweger spectrum disorders: clinical overview and management approach inherited metabolic diseases. Orphanet J Rare Dis 10:151

Klouwer FCC, Huffnagel IC, Ferdinandusse S et al (2016) Clinical and biochemical pitfalls in the diagnosis of Peroxisomal disorders. Neuropediatrics 47:205-220

Klouwer FCC, Ferdinandusse S, van Lenthe $\mathrm{H}$ et al (2017) Evaluation of C26:0-lysophosphatidylcholine and C26:0-carnitine as diagnostic markers for Zellweger spectrum disorders. J Inherit Metab Dis 40: $875-881$

Klouwer FCC, Meester-Delver A, Vaz FM et al (2018) Development and validation of a severity scoring system for Zellweger spectrum disorders. Clin Genet 93:613-621

Ploski R, Pollak A, Muller S et al (2014) Does p.Q247X in TRIM63 cause human hypertrophic cardiomyopathy? Circ Res 114:e2-e5

Raas-Rothschild A, Wanders RJ, Mooijer PA et al (2002) A PEX6defective peroxisomal biogenesis disorder with severe phenotype in an infant, versus mild phenotype resembling Usher syndrome in the affected parents. Am J Hum Genet 70:1062-1068

Rydzanicz M, Stradomska TJ, Jurkiewicz E et al (2017 Nov) Mild Zellweger syndrome due to a novel PEX6 mutation: correlation between clinical phenotype and in silico prediction of variant pathogenicity. J Appl Genet 58:475-480

Sevin C, Ferdinandusse S, Waterham HR et al (2011) Autosomal recessive cerebellar ataxia caused by mutations in the PEX2 gene. Orphanet J Rare Dis 6:8

Stradomska TJ, Tylki-Szymańska A (1996) Examination of very long chain fatty acids in diagnosis of X-linked adrenoleukodystrophy. Pediatr Pol 71:197-201

Stradomska TJ, Tylki-Szymańska A (2009) Serum very-long-chain fatty acids levels determined by gas chromatography in the diagnosis of peroxisomal disorders in Poland. Folia Neuropathol 47:306-313

Takemoto Y, Suzuki Y, Horibe R et al (2003) Gas chromatography/mass spectrometry analysis of very long chain fatty acids, docosahexaenoic acid, phytanic acid and plasmalogen for the screening of peroxisomal disorders. Brain and Development 25: $481-487$

Tran C, Hewson S, Steinberg SJ, Mercimek-Mahmutoglu S (2014) Lateonset Zellweger spectrum disorder caused by PEX6 mutations mimicking X-linked adrenoleukodystrophy. Pediatr Neurol 51:262-265

Ventura MJ, Wheaton D, Xu M et al (2016) Diagnosis of a mild peroxisomal phenotype with next-generation sequencing. Mol Genet Metab Rep 9:75-78

Wanders RJ, Klouwer FC, Ferdinandusse S et al (2017) Clinical and laboratory diagnosis of peroxisomal disorders. Methods Mol Biol 1595:329-342

Weller S, Rosewich H, Gärtner J (2008) Cerebral MRI as a valuable diagnostic tool in Zellweger spectrum patients. J Inherit Metab Dis $31: 270-280$

Publisher's note Springer Nature remains neutral with regard to jurisdictional claims in published maps and institutional affiliations. 